\title{
ANALYTICAL MODEL AND NUMERICAL STABILITY ANALYSIS FOR FALLING LIQUID FILM REGIMES IN VERTICAL PIPES
}

\author{
JOSÉ LUIS MUÑOZ-COBO ${ }^{1}$, SUSANA M. IGLESIAS ${ }^{1,2}$, DANY S. DOMINGUEZ ${ }^{1,2}$, \\ ALBERTO ESCRIVÁ $^{1}$ \& CÉSAR BERNA ${ }^{1}$ \\ ${ }^{1}$ Institute for Energy Engineering, Universitat Politècnica de València, Spain \\ ${ }^{2}$ Exact and Technological Sciences Department, Universidade Estadual de Santa Cruz, Brazil
}

\begin{abstract}
Gravity-driven falling film flows in vertical pipes are relevant in many industrial processes like evaporators, chemical reactors, and condensers. The wave formation and propagation processes, which continuously take place in the film, have a strong influence on the flow hydrodynamics and the heat and mass transfer phenomena. Several researchers have been studying the wave behaviour in these multiphase regimes through experimental works and computational fluid dynamics simulation techniques. In some simplified cases, like high viscosity fluids and infinity inclined plates, analytical solutions have been obtained. In this work, we propose an analytical model for falling film flow regimes in vertical pipes. Starting from the incompressible axisymmetric Navier-Stokes equations in cylindrical coordinates, we consider the force balance in the fluid, an asymptotic long-wave approximation and the first-order perturbation approximation for axial velocity. From this balance, we obtain a partial differential equation that describes the interface behaviour through the film thickness. The resulting equation can be solved using a numerical approach. The main resulting equation represents a stiff problem, thus, we perform a stability analysis using the fluid viscosity as a parameter. Finally, we set the model validity conditions and suggest some actions to improve the numerical strategy in order to better describe low viscosity fluids.
\end{abstract}

Keywords: falling liquid film, analytical model, vertical pipes, Navier-Stokes equations.

\section{INTRODUCTION}

The dynamics of core-annular flows are relevant in many industrial and biological applications, such as oil recovery, nuclear power plants and human body's airways. In these regimes, one fluid phase fills the core, and the other, more viscous, occupies the annulus surrounding the pipe wall. When core-phase is air, motionless or with small velocity, the volume of annular-phase is small if compared with the pipe dimensions and only the gravity force acts on the fluid, then we have a gravity-driven falling film. In addition, films flowing down on vertical pipes are very unstable and develop nonlinear waves in the interface surface. These waves have a strong influence in the flow hydrodynamics, enhancing the heat and mass transfer phenomena.

Several researchers have been studying the wave behaviour in these multiphase regimes using experimental, analytical and computational fluid dynamics (CFD) approaches. For example, Mascarenhas and Mudawar [1] using experimental and CFD techniques to study the falling heated water film in turbulent regimes, analyzing the influence of interfacial waves on mass, momentum and heat transfer. Camassa et al. [2], conducted experiments and constructed an analytical model to study the mechanism by which forced steady airflow transports annular layers of high-viscosity fluids (low Reynolds), conditions corresponding to lung airways. The model developed shows the different mass transport regimes in the wave propagation. Also, Camassa et al. [3] use a numerical model to predict the speed and the wavelength of the most unstable mode of these viscous film flows on small pipes in gravitydriven conditions. The model results are validated using experimental measurements. The 
direct numerical simulation (DNS) of the full Navier-Stokes equations, was used in [4], to study the interfacial instabilities in thin-film flows. This approach is difficult to perform due to the complexity of free surface and the needed of extremely finer meshes in space and time. Muñoz-Cobo et al. [5], applies the perturbation theory, the long-wave approximation and the velocity expansion in harmonic functions to generate an analytical model for gravity-driven film flow on inclined plates. The present work extends this work, proposing an analytical model for falling films flows regimes in vertical pipes. To construct the model, we consider the force balance in the fluid, an asymptotic long-wave approximation and the first-order perturbation approximation for axial velocity. From this balance, we obtain a partial differential equation that describes the interface behavior through the film.

At this point, we discretized the problem using a uniform spatial grid. Then, we integrate the interface equation in one arbitrary spatial cell, obtaining an ordinary differential equations system (ODES), for the film thickness at each grid cell. The resulting ODES is time integrated using an Adams-Moulton type semi-implicit predictor-corrector method to generate the numerical results. The resulting model presents a high level of stiffness leading to numerical instabilities in some conditions. At the end of this work, we perform numerical experiments using fluid viscosity as a parameter to set the model validity range.

This paper is arranged as follows. In the next section, we offer the mathematical and physical foundations of the proposed analytical model. Section 3 describes the numerical procedure and the algorithm for solving the model. In Section 4, we present the numerical experiments results and finally, in Section 5 we offer the research conclusions and suggestions for future developments.

\section{MATHEMATICAL MODEL FORMULATION}

In order to develop the analytical model for falling films in vertical pipes, we consider an axisymmetric cylindrical domain with a down annular flow regime as shown in Fig. 1(a).

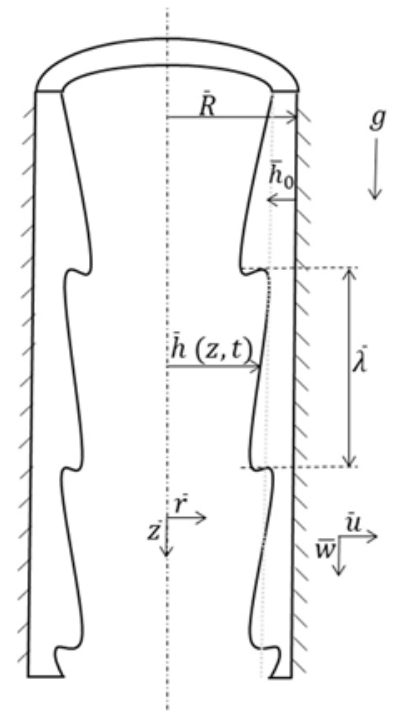

a

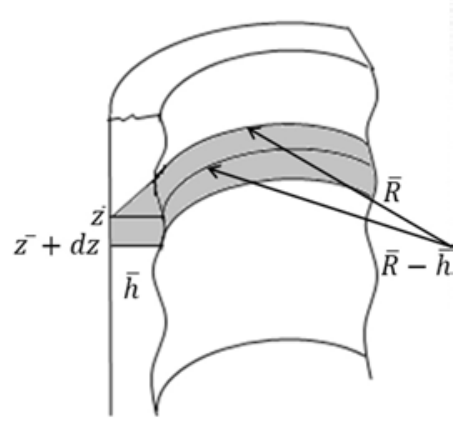

b

Figure 1: (a) Problem domain representation with the main variables; and (b) Detail of a differential film volume. 
As a starting point, we consider the incompressible axisymmetric Navier-Stokes equations in cylindrical coordinates in the form

$$
\begin{gathered}
\bar{r}^{-1} \partial_{\bar{r}}(\bar{r} \bar{u})+\partial_{\bar{z}} \bar{w}=0, \\
\partial_{\bar{t}} \bar{u}+\bar{u} \partial_{\bar{r}} \bar{u}+\bar{w} \partial_{\bar{z}} \bar{u}=-\frac{1}{\rho} \partial_{\bar{r}} \bar{P}+v\left(\frac{1}{\bar{r}} \partial_{\bar{r}}\left(\bar{r} \partial_{\bar{r}} \bar{u}\right)+\partial_{\bar{z} \bar{z}} \bar{u}-\frac{\bar{u}}{\bar{r}^{2}}\right), \\
\partial_{\bar{t} \bar{w}}+\bar{u} \partial_{\bar{r}} \bar{w}+\bar{w} \partial_{\bar{z}} \bar{w}=-\frac{1}{\rho} \partial_{\bar{z}} \bar{P}+v\left(\frac{1}{\bar{r}} \partial_{\bar{r}}\left(\bar{r} \partial_{\bar{r}} \bar{w}\right)+\partial_{\bar{z} \bar{z}} \bar{w}\right)+g,
\end{gathered}
$$

where $\bar{r}$ and $\bar{z}$ are the radial and axial coordinates, respectively; $\bar{u}$ is the radial velocity component; $\bar{w}$ is the downward direction velocity component; $\bar{P}$ is the pressure; finally, $\rho$, $v$, and $g$ indicates the fluid density, fluid kinematic viscosity and gravity acceleration, respectively. Besides, in Fig. 1(a) $\bar{h}(z, t)$ indicates the film thickness, and $\bar{h}_{0}$ is the Nusselt thickness of the flat film [6]. In our notation, the overbars denote dimensional quantities. At the pipe wall, we consider no-slip boundary conditions

$$
\left.\bar{W}\right|_{\bar{R}}=\left.\bar{u}\right|_{\bar{R}}=0 .
$$

In order to obtain the free surface boundary conditions, we regard some physical conditions at the interface. For the normal stress component, we consider that the sum of forces at the interface is zero and we obtain

$$
\begin{gathered}
\left.\bar{P}\right|_{\bar{R}-\bar{h}}-\bar{P}_{c}+\sigma\left\{\frac{\left(\partial_{\bar{z} \bar{z}} \bar{h}\right)}{\left(1+\left(\partial_{\bar{z}} \bar{h}\right)^{2}\right)^{\frac{3}{2}}}+\frac{1}{\bar{R}-\bar{h}}\right\}_{\bar{R}-\bar{h}} \\
-\frac{2 \mu}{1+\left(\partial_{\bar{z}} \bar{h}\right)^{2}}\left\{\partial_{\bar{r}} \bar{u}+\left(\partial_{\bar{z}} \bar{h}\right)\left(\partial_{\bar{z}} \bar{u}+\partial_{\bar{r}} \bar{w}\right)+\left(\partial_{\bar{z}} \bar{h}\right)^{2} \partial_{\bar{z} \bar{w}}\right\}_{\bar{R}-\bar{h}}=0,
\end{gathered}
$$

here $\bar{P}_{c}$ is the annular-phase pressure, $\sigma$ and $\mu$ indicate the fluid surface tension and the fluid dynamic viscosity respectively. The eqn (5) is similar to Young-Laplace equation [7]. Then, for the tangential stress component, we use the stress continuity condition [3] at the interface to get

$$
\left[1-\left(\partial_{\bar{z}} \bar{h}\right)^{2}\right]\left\{\partial_{\bar{z}} \bar{u}+\partial_{\bar{r}} \bar{w}\right\}_{\bar{R}-\bar{h}}+2 \partial_{\bar{z}} \bar{h}\left\{\partial_{\bar{z}} \bar{w}-\partial_{\bar{r}} \bar{u}\right\}_{\bar{R}-\bar{h}}=0
$$

We can obtain an equation to govern the interface using a film mass balance in the interval $[z, z+d z]$. The geometry of the mass balance is displayed in Fig. 1(b). Considering that the mass flow variation over time in the film differential volume is equal to the difference between the flow entering by the top surface and the flow leaving by the bottom surface, then we have

$$
\partial_{\bar{t}}\left[2 \pi \rho \bar{h}\left(\bar{R}-\frac{\bar{h}}{2}\right)\right]=-\partial_{\bar{z}}\left[\rho \int_{\bar{R}-\bar{h}}^{\bar{R}} 2 \pi \bar{r} \bar{w} d \bar{r}\right] .
$$

Solving the integral in eqn (7) using the Leibnitz rule and considering the mass conservation eqn (1), we obtain

$$
\partial_{\bar{t}} \bar{h}(\bar{z}, \bar{t})=-\left[\left.\bar{w}\right|_{\bar{R}-\bar{h}} \partial \bar{z} \bar{h}+\left.\bar{u}\right|_{\bar{R}-\bar{h}}\right]
$$


The eqn (8) is a partial differential equation that describes the fluid interface as a function of the time and z-coordinate, some authors call this equation, a kinematic boundary condition [3]. Is possible to obtain the integral formulation for the interface equation. For that, we define $\bar{q}$ as the mass flow rate through a radian of the circumference in the form

$$
\bar{q}=\int_{\bar{R}-\bar{h}}^{\bar{R}} \bar{r} \bar{w}(\bar{r}, \bar{z}, \bar{t}) d \bar{r},
$$

then, we calculate the time derivative on the eqn (7) left side. Considering eqn (9) for the right side and grouping, the interface integral formulation is written as

$$
(\bar{R}-\bar{h}) \partial_{t} \bar{h}+\partial_{\bar{z}} \bar{q}=0 .
$$

At this stage, we introduce the dimensionless parameters to transform the equations system into a non-dimensional form. This dimensionless scheme does not depend on mass flow quantities and was proposed by Ruyer-Quil and Manneville [8]. The dimensionless parameters are

$$
L_{c}=\left(\frac{v^{2}}{g}\right)^{1 / 3}, T_{c}=\left(\frac{v}{g^{2}}\right)^{1 / 3}, U_{r}=(v g)^{1 / 3}, P_{r}=\rho(v g)^{2 / 3}, K_{a}=\frac{\sigma}{\rho v^{4 / 3} g^{1 / 3}},
$$

where $L_{c}$ and $T_{c}$ are the characteristic longitude and time, $U_{r}$ and $P_{r}$ are the reference velocity and pressure and $K_{a}$ is the Kapitza number. The mass conservation and momentum eqns (1)(3) in the dimensionless form are written as

$$
\begin{gathered}
r^{-1} \partial_{r}(r u)+\partial_{z} w=0 \\
\partial_{t} u+u \partial_{r} u+w \partial_{z} u=-\partial_{r} P+r^{-1} \partial_{r}\left(r \partial_{r} u\right)+\partial_{z z} u-\frac{u}{r^{2}} \\
\partial_{t} w+u \partial_{r} w+w \partial_{z} w=-\partial_{z} P+r^{-1} \partial_{r}\left(r \partial_{r} w\right)+\partial_{z z} w+1 .
\end{gathered}
$$

The wall boundary condition (eqn (4)), appear as

$$
\left.w\right|_{R}=\left.u\right|_{R}=0 \text {, }
$$

and the boundary conditions at the fluid interface (eqns (5) and (6)) become

$$
\begin{gathered}
\left.P\right|_{R-h}-P_{c}+K a\left\{\frac{\partial_{z z} h}{\left(1+\left(\partial_{z} h\right)^{2}\right)^{\frac{3}{2}}}+\frac{1}{R-h}\right\}_{R-h} \\
-\frac{2}{1+\left(\partial_{z} h\right)^{2}}\left\{\partial_{r} u+\left(\partial_{z} h\right)\left(\partial_{z} u+\partial_{r} w\right)+\left(\partial_{z} h\right)^{2} \partial_{z} w\right\}_{R-h}=0, \\
{\left[1-\left(\partial_{z} h\right)^{2}\right]\left\{\partial_{z} u+\partial_{r} w\right\}_{R-h}+2 \partial_{z} h\left\{\partial_{z} w-\partial_{r} u\right\}_{R-h}=0 .}
\end{gathered}
$$

The PDE interface equation (eqn (8)) and the corresponding integral form (eqn (10)) are transformed into

$$
\begin{gathered}
\partial_{t} h(z, t)=-\left[w_{R-h} \partial z h+\left.u\right|_{R-h}\right] \\
(R-h) \partial_{t} h+\partial_{z} q=0
\end{gathered}
$$

respectively. In eqn (19), the parameter $q$ adopts the non-dimensional form of eqn (9) 


$$
q=\int_{R-h}^{R} r w(r, z, t) d r .
$$

The interface integral eqn (19) relates the film thickness $h$, with the axial velocity $w$. Then, if we obtain one approximation for the axial velocity, we can evaluate the integral term in eqn (20) and solve the resultant PDE to get the film thickness.

Now, we regard the long-wave approach [3], [5], [6], this is, when the film surface perturbations wavelength $(\lambda)$, is greater than the film thickness. Thus, the perturbation parameter is defined by

$$
\varepsilon=\frac{h}{\lambda} \ll 1
$$

Rewriting the constitutive eqns (12)-(14), the corresponding boundary conditions eqns (15)-(17) and the interface eqn (19) and considering a long-wave approximation, we generate the general perturbative equations. Here, the terms involving temporal and z-spatial derivatives, are first order in $\varepsilon$.

From these considerations, the resulting balance equations are

$$
\begin{gathered}
r^{-1} \partial_{r}(r u)+\varepsilon \partial_{z} w=0 \\
\varepsilon \partial_{t} u+u \partial_{r} u+\varepsilon w \partial_{z} u=-\partial_{r} P+r^{-1} \partial_{r}\left(r \partial_{r} u\right)+\varepsilon^{2} \partial_{z z} u-\frac{u}{r^{2}} \\
\varepsilon \partial_{t} u+u \partial_{r} u+\varepsilon w \partial_{z} u=-\varepsilon \partial_{z} P+r^{-1} \partial_{r}\left(r \partial_{r} w\right)+\varepsilon^{2} \partial_{z z} w+1 .
\end{gathered}
$$

The perturbative equations for the normal (eqn (16)) and tangential (eqn (17)) stress at the film surface are written as

$$
\begin{gathered}
\left.P\right|_{R-h}-P_{c}+K a\left\{\frac{\varepsilon^{2} \partial_{z z} h}{\left[1+\varepsilon^{2}\left(\partial_{z} h\right)^{2}\right]^{\frac{3}{2}}}+\frac{1}{R-h}\right\}_{R-h} \\
-\frac{2}{1+\varepsilon^{2}\left(\partial_{z} h\right)^{2}}\left\{\partial_{r} u+\varepsilon\left(\partial_{z} h\right)\left(\varepsilon \partial_{z} u+\partial_{r} w\right)+\varepsilon^{3}\left(\partial_{z} h\right)^{2} \partial_{z} w\right\}_{R-h}=0, \\
{\left[1-\varepsilon^{2}\left(\partial_{z} h\right)^{2}\right]\left\{\varepsilon \partial_{z} u+\partial_{r} w\right\}_{R-h}+2 \varepsilon \partial_{z} h\left\{\varepsilon \partial_{z} w-\partial_{r} u\right\}_{R-h}=0 .}
\end{gathered}
$$

For the wall boundary condition in eqn (15) and the interface eqn (19), their perturbative form is equal to the corresponding dimensionless equations. At this point, we have all the equations in the perturbative mode. It is possible to find the problem's solutions, this is, velocities and pressure, using the first-order expansion expresses as

$$
\begin{aligned}
& u=u_{0}+\varepsilon u_{1}, \\
& w=w_{0}+\varepsilon w_{1}, \\
& p=p_{0}+\varepsilon p_{1} .
\end{aligned}
$$

Now, we write the zero-order perturbative equations. To perform this step, we substitute the expansion eqns (27)-(29) in the perturbative eqns (22)-(26) and (15) and take the limit $\varepsilon \rightarrow 0$.

For the continuity eqn (22) we obtain 


$$
r^{-1} \partial_{r}\left(r u_{0}\right)=0
$$

here, considering the wall boundary conditions, we have that $u_{0}(r)=0$. This result is consistent with the problem phenomenology where the changes in the radial direction are lower in order than the changes in the axial direction. For the momentum eqns (23) and (24) and considering the previous result for $u_{0}$, we have

$$
\begin{gathered}
\partial_{r} P_{0}=0, \\
\bar{r}^{-1} \partial_{r}\left(r \partial_{r} w_{0}\right)=-1,
\end{gathered}
$$

for the normal stress eqn (25) we obtain

$$
\left.P_{0}\right|_{R-h}=P_{c}-K a\left\{\partial_{z z} h+\frac{1}{R-h}\right\}_{R-h},
$$

here, we use a serial expansion to retain the axial curvature term according with [9]. For the tangential stress eqn (26) the results is

$$
\left.\partial_{r} w_{0}\right|_{R-h}=0,
$$

and the non-slip wall boundary conditions appear as

$$
\left.w_{0}\right|_{R}=\left.u_{0}\right|_{R}=0 .
$$

We integrate the eqn (32) to obtain the zero-order moment for radial velocity $w_{0}$,

$$
w_{0}(r, z)=\frac{(R-h)^{2}}{2} \log \left(\frac{r}{R}\right)+\frac{\left(R^{2}-r^{2}\right)}{4},
$$

finally, using eqn (31) and the respective boundary condition (33), we have $P_{0}$ expressed as

$$
P_{0}(z, t)=P_{c}-K a\left\{\partial_{z z} h+\frac{1}{R-h}\right\}
$$

At this point we have the zero-order equations and their corresponding solutions. Now, we proceed with the first-order formulation.

We substitute the first-order expansions (27)-(29) in eqns (22)-(26) and (15) and consider the first order terms, to obtain the first order constitutive equations. So, the first order continuity equation appears as

$$
r^{-1} \partial_{r}\left(r u_{1}\right)+\partial_{z} w_{0}=0
$$

in (38), we use eqn (36) for $w_{0}$ and integrate to obtain $u_{1}$ in the form

$$
u_{1}(r, z, t)=\frac{\partial_{z} h}{r}(R-h)\left\{\frac{r^{2}}{2} \log \left(\frac{r}{R}\right)+\frac{R^{2}-r^{2}}{4}\right\} .
$$

The corresponding first-order radial momentum equation appear as

$$
\partial_{r} P_{1}=\bar{r}^{-1} \partial_{r}\left(r \partial_{r} u_{1}\right)-\frac{u_{1}}{r^{2}}
$$

the first-order axial momentum equation is written in the form

$$
\bar{r}^{-1} \partial_{r}\left(r \partial_{r} w_{1}\right)=\partial_{z} P_{0}+\partial_{t} w_{0}+u_{1} \partial_{r} w_{0}+w_{0} \partial_{z} w_{0},
$$

at the interface the first-order tangential stress equation is written as 


$$
\left.\partial_{r} w_{1}\right|_{R-h}=0,
$$

and the boundary condition at wall

$$
\left.w_{1}\right|_{R}=0 .
$$

Using the solutions for $P_{0}$, (eqn (37)), $w_{0}$ (eqn (36)) and $u_{1}$ (eqn (39)), we integrate the eqn (41) to obtain the first-order component of radial velocity $\left(w_{1}\right)$. After some algebraic work, the result appears as

$$
\begin{aligned}
& w_{1}(r, z, t)= K_{a j} \partial_{z z z} h\left\{\frac{R^{2}}{4}-\frac{r^{2}}{4}+\frac{(R-h)^{2}}{2} \log \left(\frac{r}{R}\right)\right\}+ \\
& K_{a} \partial_{z} h\left\{\frac{1}{2} \log \left(\frac{r}{R}\right)+\frac{R^{2}}{4(R-h)^{2}}-\frac{r^{2}}{4(R-h)^{2}}\right\}+ \\
& \frac{\partial_{z} h}{128}(R-h)\left\{r^{4}+11 R^{4}-12 r^{2} R^{2}+8 r^{2} R^{2} \log \left(\frac{r}{R}\right)\right\}+ \\
& \frac{\partial_{z} h}{32}(R-h)^{3}\left\{5 R^{2}-5 r^{2}+\left(6 r^{2}+4 R^{2}\right) \log \left(\frac{r}{R}\right)-\right. \\
&\left(r^{2}-R^{2}\right)\left[8 \log \left(\frac{R-h}{R}\right)\right]-4 r^{2} \log ^{2}\left(\frac{r}{R}\right)+ \\
&\left.\log \left(\frac{r}{R}\right) \log \left(\frac{R-h}{R}\right)\left(8 r^{2}-4 R^{2}\right)\right\} \\
& \frac{\partial_{z} h}{16}(R-h)^{3}\left\{R^{2}[\log (r)-\log (R)]-2 R^{2} \log ^{2}\left(\frac{r}{R}\right) \log (R-h)\right\}+ \\
& \frac{\partial_{z} h}{8}(R-h)^{5}\left\{\log ^{2}\left(\frac{r}{R}\right)\left[\log ^{2}\left(\frac{R-h}{R}\right)-2 \log ^{2}\left(\frac{R-h}{R}\right)+\frac{3}{4}\right]\right\} .
\end{aligned}
$$

Eqns (36) and (44) represent the first-order approximation for radial velocity. Using these approximations in the interface integral eqn (19) we can solve it, through a numerical procedure obtaining the liquid interface behavior. In the next section, we describe the numerical approach.

\section{NUMERICAL PROCEDURE}

To analyze the film hydrodynamics, specifically the wave evolution under different geometrical and physical conditions, we have developed a numerical method to integrate the eqn (19). We set the calculation domain to a test pipe with $0 \leq z \leq L$ of length. Firstly, we discretize this domain using a uniform grid of $N$ spatial cells, with a length of $\Delta z=L / N$. The spatial cell boundaries are given by $z_{i}=(i-1) \Delta z$, with $i=1: N+1$. The coordinates of each spatial cell were taken at the spatial cell centers and denoted by overdot, i.e. $\dot{z}_{i}=(i-1 / 2) \Delta z$ with $i=1: N$. So, an overdot in a magnitude with sub-index $i$ means that magnitude is being calculated at the center of the $i$-th spatial cell, located between the boundaries $z_{i}$ and $z_{i+1}$. In the other hand, a magnitude with sub-index $i$ without overdot is related to the i-th cell boundary.

In the second step, we spatially discretize the PDE (eqn (19)). Then, we apply the averaging operator over a spatial cell 


$$
\dot{f}_{i}=\frac{1}{\Delta z} \int_{z_{i}}^{z_{i+1}} f(z) d z
$$

in eqn (19). The result is written as

$$
d_{t} \dot{h}_{i}(t)=-\frac{q_{i+1}(t)-q_{i}(t)}{\left[R-\dot{h}_{i}(t)\right] \Delta z}, \quad i=1 \ldots N .
$$

here the mass flow by a radian values $q_{i}(t)$ are obtained integrating eqn (20) over the film and evaluating for the corresponding $z_{i}$. To integrate eqn (20), we use the approximation for radial velocity defined by eqns (36) and (44). The first and third z-derivatives that appears in $q_{i}(t)$ are numerically evaluated at the boundaries of each spatial cell using an appropriate difference scheme [10].

The ordinary differential equation system represented by eqn (46) has one equation per spatial cell with the corresponding average film thickness $\dot{h}_{i}(t)$ as unknown. To express the film thickness at the cell boundaries $h_{i}(t)$ in terms of the values at the node centers, we consider a linear approximation in the form

$$
h_{i}(t)=\left(\dot{h}_{i-1}(t)+\dot{h}_{i}(t)\right) / 2, \quad i=2 \ldots N,
$$

for the boundaries $h_{1}(t)$ and $h_{N+1}(t)$ values, we derive appropriate linear schemes using the left/right cell centers values. The linear approximation is a good approach for a fine uniform spatial grid [11].

The coefficients analysis and the preliminary calculations show that the ODE system (46) is a stiff problem [12]. A strategy to reduce the stiffness is to decrease the coefficients order, in consequence, we manipulate (46), extracting $R^{5}$ factor in the right side numerator, and introducing a variable change $\dot{h}_{i}(t)=\dot{h}_{i}^{\prime}(t) / R$, the result is

$$
d_{t} \dot{h}_{i}^{\prime}(t)=-\frac{R^{3}\left[q_{i+1}^{\prime}(t)-q_{i}^{\prime}(t)\right]}{\left[1-\dot{h}_{i}^{\prime}(t)\right] \Delta z} .
$$

To solve the system of eqn (48), we use a semi-implicit fourth order Adams-Moulton predictor-corrector method, with the prediction step based on the Adams-Bashforth algorithm [5], [12]. To initialize the Adams-Moulton multi-step method, we perform four iterations using a classical Runge-Kutta method [11]. As the initial condition for the problem, we use a flat Nusselt profile in all domain, adding at the first-quarter domain inlet, a small multiple modes Fourier perturbation [2] expressed as

$$
h(z, 0)=h_{0}-\sum_{k=1}^{K} b \cos \left(2 \pi k z+\alpha_{k}\right), \quad 0 \leq z \leq L / 4,
$$

where $K$ is the Fourier modes number, typically $K=6$, the amplitude $b$ satisfy $\left(b<0.05 h_{0}\right)$, and $\alpha_{k}$ is a random phase shift for each mode.

\section{NUMERICAL RESULTS}

To evaluate the analytical model and numerical procedure stability, we select a one-wave problem, this is, we study the initial perturbation over the pipe without introducing new inlet perturbations. We perform simulations for six different fluids and observe the solution stability. The selected fluids and their properties are listed in Table 1. Preliminary simulations 
showed that the stability is directly related to the fluid viscosity, for that reason we choose fluids with viscosities varying from $1 \mathrm{E}-2$ to $1 \mathrm{E}-7$.

Table 1: Fluid types and physical properties used in numerical experiments.

\begin{tabular}{|l|c|c|c|c|}
\hline Fluid & $\mathrm{T}\left({ }^{\circ} \mathrm{C}\right)$ & $v\left(\mathrm{~m}^{2} / \mathrm{s}\right)$ & $\rho\left(\mathrm{kg} / \mathrm{m}^{3}\right)$ & $\sigma(\mathrm{N} / \mathrm{m})$ \\
\hline Water & 25 & $8.930 \mathrm{E}-07$ & $9.970 \mathrm{E}+02$ & $7.200 \mathrm{E}-02$ \\
\hline Fuel oil light & 20 & $1.650 \mathrm{E}-05$ & $9.100 \mathrm{E}+02$ & $2.300 \mathrm{E}-02$ \\
\hline Castor oil & 30 & $5.800 \mathrm{E}-04$ & $9.550 \mathrm{E}+02$ & $3.900 \mathrm{E}-02$ \\
\hline Glycerine & 20 & $1.183 \mathrm{E}-03$ & $1.261 \mathrm{E}+03$ & $6.340 \mathrm{E}-02$ \\
\hline Fuel oil heavy & 20 & $8.000 \mathrm{E}-03$ & $9.900 \mathrm{E}+02$ & $2.300 \mathrm{E}-02$ \\
\hline Silicone oil [3] & - & $1.330 \mathrm{E}-02$ & $9.700 \mathrm{E}+02$ & $2.150 \mathrm{E}-02$ \\
\hline
\end{tabular}

The selected simulation domain is a pipe with $2.1 \mathrm{E}-2 \mathrm{~m}$ radius and $2.5 \mathrm{~m}$ length. The initial Nusselt thickness corresponds to $1 \mathrm{E}-3 \mathrm{~m}$ and the initial perturbation amplitude was $b=0.03$. With this domain, we perform a mesh optimization study. Thus, empirically determine the maximum space and temporal steps $(\Delta z$ and $\Delta t)$, with which we can solve the problem without significant result differences for thinner meshes. For the spatial mesh the optimum step for all the fluids was $\Delta z=1 E-3 m$. The temporal step for silicone oil, fuel oil heavy, glycerin, and castor oil was $\Delta t=5.0 E-5 \mathrm{~s}$ while for fuel oil light and water was $\Delta t=5.0 E-7 s$.

Fig. 2. shows the wave temporal evolution for castor oil fluid. At the time $t=0$, the initial condition, a multiple cosinusoidal perturbation is observed in the domain first quarter, being its maximum amplitude about $1.15 h_{0}$. In the final three quarters of this profile, we have a flat behavior. Observing the time evolution we can notice that after the first seconds, the wave begin to change its shape, compressing and increasing its height, until it reach the maximum

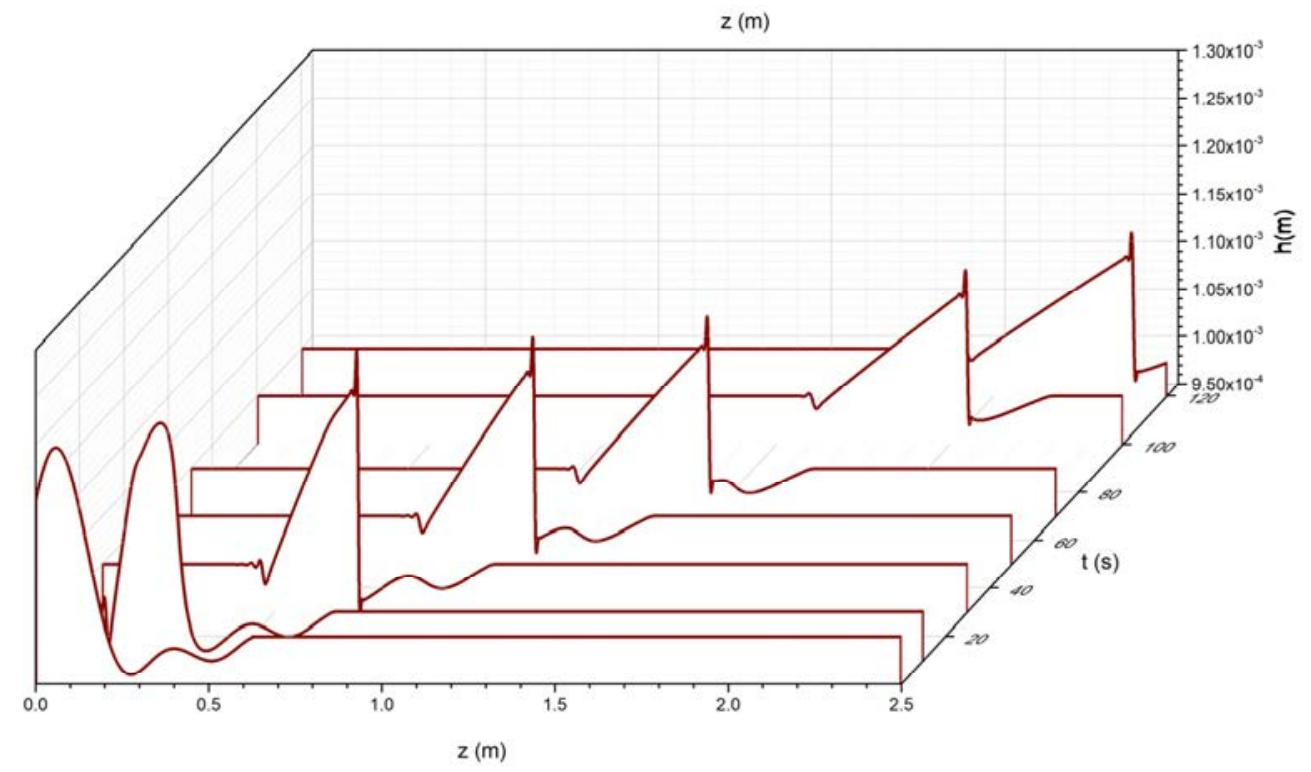

Figure 2: Temporal wave evolution for the castor oil fluid. 
$\left(1.25 h_{0}\right.$ approximately at $\left.t_{m}=25 \mathrm{~s}\right)$. From the maximum amplitude point, the wave holds its shape, and the amplitude slowly decrease until $t_{s}=115 \mathrm{~s}$ and $z_{s}=2.3 \mathrm{~m}$ position. Next, these values stay stationary until the wave leaves the domain. Thus, the wave evolution reached the steady state.

The wave qualitative behavior of the others evaluated fluids is similar, varying the domain position and time where the wave becomes steady. In Table 2 we show the amplitude, position and time were the wave reaches the maximum $\left(h_{m}, z_{m}, t_{m}\right)$. In addition, in Table 3 we offer this values for the steady state $\left(h_{s}, z_{s}, t_{s}\right)$ within the time when the wave leaves the domain $\left(t_{T}\right)$. Finally, Fig. 3 shows the fluid interfaces in the steady state beginning and data as film thickness and domain positions are offered.

Table 2: Wave evolution features for study fluids at maximum amplitude.

\begin{tabular}{|l|c|c|c|}
\hline Fluid & $h_{m}(\mathrm{~m})$ & $z_{m}(\mathrm{~m})$ & $t_{m}(\mathrm{~s})$ \\
\hline Castor oil & $1.236 \mathrm{E}-03$ & $5.895 \mathrm{E}-01$ & $2.250 \mathrm{E}+01$ \\
\hline Glycerine & $1.247 \mathrm{E}-03$ & $5.710 \mathrm{E}-01$ & $4.400 \mathrm{E}+01$ \\
\hline Fuel oil heavy & $1.237 \mathrm{E}-03$ & $5.770 \mathrm{E}-01$ & $2.980 \mathrm{E}+02$ \\
\hline Silicone oil & $1.238 \mathrm{E}-03$ & $5.930 \mathrm{E}-01$ & $5.520 \mathrm{E}+03$ \\
\hline
\end{tabular}

Table 3: Wave evolution features for study fluids at reach steady stage, and total time.

\begin{tabular}{|l|c|c|c|c|}
\hline Fluid & $h_{S}(\mathrm{~m})$ & $z_{S}(\mathrm{~m})$ & $t_{S}(\mathrm{~s})$ & $t_{T}(\mathrm{~s})$ \\
\hline Castor oil & $1.122 \mathrm{E}-03$ & $2.290 \mathrm{E}+02$ & $1.150 \mathrm{E}+02$ & $1.540 \mathrm{E}+02$ \\
\hline Glycerine & $1.147 \mathrm{E}-03$ & $1.861 \mathrm{E}+00$ & $1.830 \mathrm{E}+02$ & $3.120 \mathrm{E}+02$ \\
\hline Fuel oil heavy & $1.179 \mathrm{E}-03$ & $1.430 \mathrm{E}+00$ & $7.550 \mathrm{E}+02$ & $1.840 \mathrm{E}+03$ \\
\hline Silicone oil & $1.183 \mathrm{E}-03$ & $1.293 \mathrm{E}+00$ & $1.489 \mathrm{E}+03$ & $3.251 \mathrm{E}+03$ \\
\hline
\end{tabular}

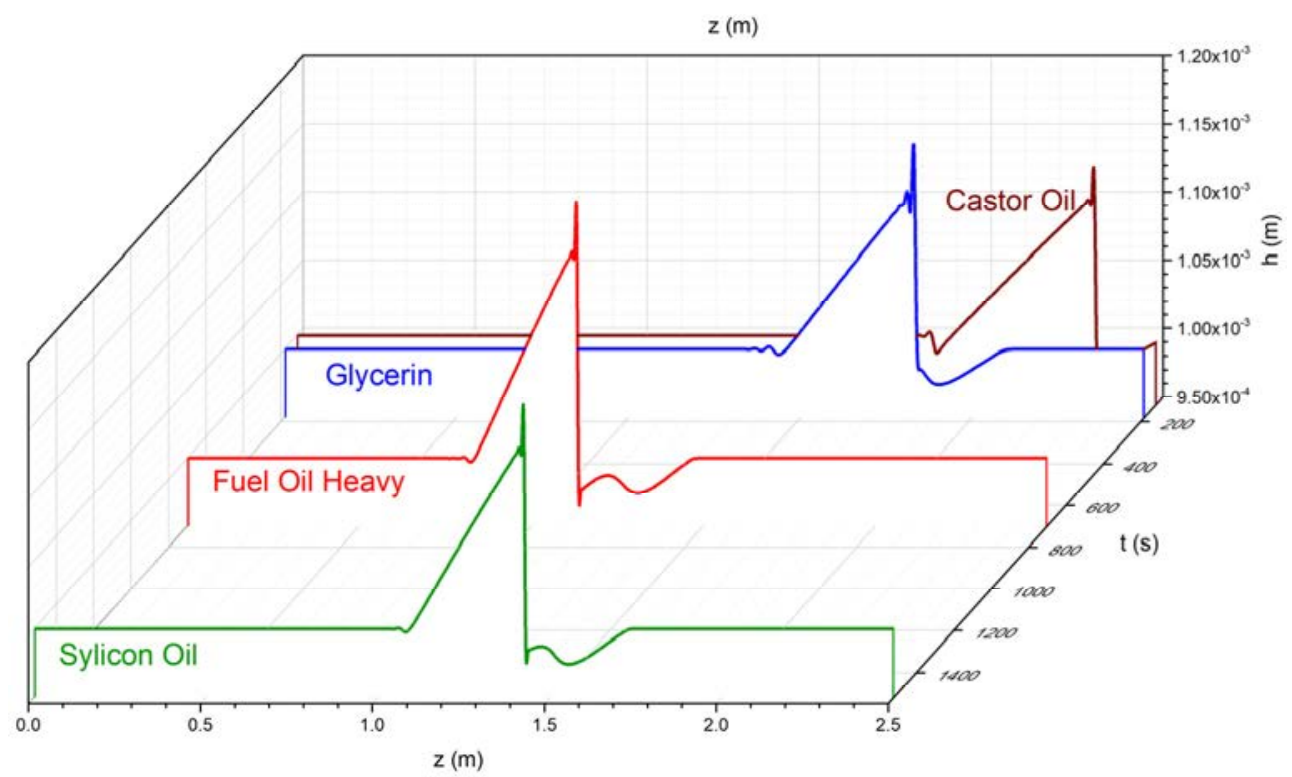

Figure 3: Fluid profiles when the wave evolution reach steady state. 
The solutions for water and fuel oil light showed up unstable, for that reason, this data is not reported in Fig. 3 and Tables 2 and 3. For these low viscosity fluids, the algorithm results were not satisfactory.

Table 2 shows that the maximum wave height and its position do not depend on the fluid, being governed only by geometry and initial conditions. However, the time taken for the wave deformation, and the time needed to reach the maximum amplitude increase considerably with the fluid viscosity

Analyzing the Table 3 and Fig. 3, we can verify that when the fluid viscosity increases, the distance travelled by the wave to become steady decreases, while increasing the maximum wave height in steady state. Moreover, the time to reach this state and for the wave leaves the pipe, are significantly higher with the viscosity increment. The wave evolution in each fluid and the behavior dependence on kinetic viscosity, is consistent with the problem physic.

\section{CONCLUSIONS AND FUTURE WORKS}

In this work, we present an analytical model to simulate the wave evolution in gravity driven falling films in vertical pipes. The model is represented by an integral-differential equation that describes the film thickness as a function of the axial velocity.

The proposed numerical strategy discretizes the problem and solves the resultant ODE system using a semi-implicit fourth order predictor-corrector method. The analytical model and numerical procedure were evaluated solving a one-wave problem for fluids with different viscosities.

The model and numerical approach offered satisfactory results for medium and high viscosity fluids using fine temporal and spatial meshes. Nevertheless, for low viscosity fluids was not possible to reproduce the wave evolution, being the results unstable. The ODE system associated with this model is stiff, feature that is highlighted for low viscosity fluids. In this situation, the proposed numerical procedure is not appropriate.

In the short-term, this research future steps involve more robust numerical strategies, recommended for high stiffness problems, and techniques to evaluate the ODE's numerical stability. Therefore, we are expecting to get a numerical procedure capable to solve the model for low viscosity fluids with a good performance using thick spatial and temporal steps.

As a long-term objective, we need to evaluate the proposed model in more complex regimes involving multiple waves. In addition, we will use available experimental results to validate the model.

\section{ACKNOWLEDGEMENTS}

The authors are indebted to the plan of I+D support of the EXMOTRANSIN project ENE2016-79489-C2-1-P. Authors, Susana M. Iglesias and Dany S. Dominguez, acknowledge the Universidade Estadual de Santa Cruz for supporting this research.

\section{REFERENCES}

[1] Mascarenhas, N. \& Mudawar, I., Study of the influence of interfacial waves on heat transfer in turbulent falling films. International Journal Heat Mass Transfer, 67, pp. 1106-1121, 2013.

[2] Camassa, R., Forest, M.G., Lee, L., Ogrosky, H.R. \& Olander, J., Ring waves as a mass transport mechanism in air-driven core-annular flows. Physical Review E, 86(6), p. $66305,2012$. 
[3] Camassa, R., Ogrosky, H.R. \& Olander, J., Viscous film flow coating the interior of a vertical tube. Part 1: Gravity-driven flow. Journal of Fluid Mechanics, 745, pp. 682715, 2014.

[4] Ramaswamy, B., Chippada, S. \& Joo, S., A full-scale numerical study of interfacial instabilities in thin-film flows. Journal of Fluid Mechanics, 325, pp. 163-194, 1996.

[5] Muñoz-Cobo, J.L., Miquel, A., Berna, C. \& Escrivà, A., Spatial and time evolution of non linear waves in falling liquid films by the harmonic expansion method with predictor-corrector integration. Proceedings of the 12th International Conference on Heat Transfer, Fluid Mechanics and Thermodynamics, pp. 91-97, 2016.

[6] Kalliadasis, S., Ruyer-Quil, C., Scheid, B. \& Velarde, M.G., Falling Liquid Films, Springer-Verlag: London, 2012.

[7] Chen, T., Chiu, M.-S. \& Weng, C.-N., Derivation of the generalized Young-Laplace equation of curved interfaces in nanoscaled solids. Journal of Applied Physics, 100(7), p. 74308, 2006.

[8] Ruyer-Quil, C. \& Manneville, P., Modeling film flows down inclined planes. European Physics Journal B, 6, pp. 277-292, 1998.

[9] Craster, R.V. \& Matar, O.K., Dynamics and stability of thin liquid films. Review of Modern Physics, 81, pp. 1131-1198, 2009.

[10] Minkowycz, W.J., Sparrow, E.M. \& Murthy, J.Y., Handbook of Numerical Heat Transfer, John Wiley \& Sons: Hoboken, NJ, 2000.

[11] Hoffman, J.D. \& Frankel, S., Numerical Methods for Engineers and Scientists, 2nd ed., Marcel Dekker: New York, 2001.

[12] Atkinson, K., Han, W. \& Stewart, D., Numerical Solution of Ordinary Differential Equations, John Wiley \& Sons: Hoboken, NJ, 2009. 